\title{
Lu pour Vous
}

\section{Clinique}

Françoise Chapon

\section{Score prédictif du risque de tachyarythmie ventriculaire dans les mutations du gène LMNA}

\section{Résumé}

La manifestation clinique la plus fréquente des laminopathies (liées à une mutation dans le gène LMNA) est la cardiomyopathie dilatée arythmogène avec risque de mort subite (MS) par survenue de tachyarythmie ventriculaire (TAV). Le traitement préventif en est la mise en place d'un défibrillateur implantable (DI). La détection des sujets à risque est ainsi indispensable.

L'objectif de cette étude [1] était d'évaluer plus précisément le risque de MS par TAV afin de définir au mieux les indications du DI.

L'étude a porté sur une base de données de 839 patients, 660 du registre national français pour constituer l'échantillon d'analyse (ramené à 444 après application des critères d'inclusion et d'exclusion) et 179 provenant de cinq centres étrangers de cardiomyopathies pour former l'échantillon de validation, l'étude concernant pour les deux échantillons des patients âgés de plus de 16 ans à la première évaluation cardiaque entre janvier 2000 et juin 2017.

La méthodologie a été celle de l'établissement d'un modèle prédictif de risque de TAV fatale ou presque fatale définie ainsi 1/MS, 2/traitement approprié par ID, 3/ autres manifestations d'une TAV instable. Huit variables prédictives ont été retenues : âge, sexe masculin, mutations autres que faux-sens, bloc AV $1^{\text {er }}$ degré, bloc AV supérieur à $1^{\text {er }}$ degré, arythmie auriculaire, tachycardie ventriculaire non soutenue, fraction d'éjection du ventricule gauche. Le modèle mathématique utilisé est le modèle de régression multivariée de Fine-Gray (validation interne sur l'échantillon d'analyse par «bootstraping » sur 100 itérations) suivi d'une validation externe en utilisant l'échantillon de validation. Pour cette dernière, deux indicateurs ont

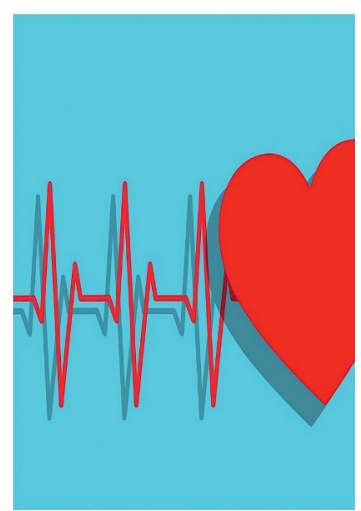

CHU de Caen-Normandie, France. Centre de Compétences des Maladies neuromusculaires, Caen France.

francoise.chapon@unicaen.fr

été utilisés dans chacun des deux échantillons: l'index de confiance (C-index) et l'étendue d'étalonnage (calibration scope).

Enfin, les auteurs ont comparé leur modèle de prédiction de risque sur cinq ans avec celui d'une évaluation suivant les recommandations de référence actuellement appliquées. Les résultats indiquent que ce nouveau score de prédiction aurait ainsi permis en comparaison du score des recommandations établies antérieurement une meilleure évaluation des risques en évitant 1 /une mort subite pour certains patients et $2 /$ une implantation non nécessaire d'un défibrillateur pour d'autres patients.

\section{Commentaire}

Étude importante qui vient préciser les critères d'évaluation des risques de mort subite chez des patients âgés de plus de 16 ans atteints de laminopathies. En effet, les recommandations de l'American College of Cardiology et de l'American Heart Association Heart Rhythm Society ainsi que de l'European Society of Cardiology s'accordent sur l'indication d'implantation d'un défibrillateur chez les patients présentant deux ou plus facteurs de risque parmi les quatre suivants: sexe masculin, mutation non faux-sens, tachycardie ventriculaire non soutenue, fraction d'éjection du ventricule gauche inférieure à $45 \%$. Les auteurs ont évalué davantage de critères et utilisé une méthode analytique très fiable pour valider leurs propos. Le reclassement des patients selon leurs critères a mis en évidence une meilleure prédiction du risque. II faut souligner l'importance des cohortes analysées grâce au caractère international de l'étude. $\diamond$ Risk prediction score for ventricular tachyarrhythmias in LMNA mutations

LIENS D'INTÉRÊT

F. Chapon fait partie des 109 co-auteurs du papier présenté.

\section{RÉFÉRENCES}

1. Wahbi K, Ben Yaou R, Gandjbakhch $\varepsilon$, et al. Development and validation of a new risk prediction score for life-threatening ventricular tachyarrhythmias in laminopathies. Circulation $2019 ; 140: 293-302$. 